\title{
Estilo parental y resiliencia en adolescentes de educación superior
}

Parenting style and resilience in adolescents of superior education

\author{
Marcia Díaz Cano Cáceres \\ Caroline Melgar Carrasco \\ Eduardo Marquina Carrasco \\ Patricia Isasa de Rondón \\ Universidad Católica San Pablo, Perú \\ Correspondencia: marcia.diazcano@ucsp.edu.pe
}

Recibido: 28-01-2017

Aceptado: 18-03-2016

\section{Resumen}

El objetivo de la presente investigación es identificar la relación entre el estilo parental y la resiliencia, variables que influyen en la deserción académica. Se tomó como muestra 201 estudiantes de edades comprendidas entre los 16 y 19 años (72 varones y 128 mujeres) de un instituto de educación superior de Arequipa. Para lo cual, se aplicaron los siguientes instrumentos: Escala para la evaluación del estilo parental, que consta de 41 ítems que identifica seis dimensiones, y la Escala de Resiliencia, que cuenta con 25 ítems con cuatro factores. Los resultados obtenidos indican que sí hay correlación entre las variables estudiadas, con ello se demuestra que los estilos parentales tienen relación directa con la resiliencia que desarrollan los adolescentes.

Palabras clave: Estilo parental, resiliencia, adolescentes.

\begin{abstract}
The aim of the present research is to identify the relationship between parenting style and resilience, variables that influence academic desertion. A sample of 201 students aged between 16 and 19 (72 males and 128 females) from a higher education institute in Arequipa was taken as a sample. For which the following instru-
\end{abstract}


ments were applied: Scale for the evaluation of the parental style that has 41 items that identifies six dimensions, and the Resilience Scale with 25 items and four factors. The results obtained indicate that there is a correlation between the studied variables, which demonstrates that the parenting styles are directly related to the resilience that adolescents develop.

Key words: Parenting style, resilience, adolescents.

A la memoria de Patricia Isasa

\section{Introducción}

Los niveles de deserción estudiantil en Perú continúan siendo un desafío para las políticas educativas y la sociedad en general. En Arequipa, se considera que, en los últimos cinco años, el $60 \%$ de estudiantes de institutos superiores ha abandonado los estudios (Lozada, 2012). Se sabe que son muchos los factores que influyen en los estudiantes al momento de abandonar sus prácticas académicas, pero, como en la mayoría de problemas, el papel de la resiliencia es fundamental (Villalta, 2009), siendo esta un conjunto de recursos útiles para superar el fracaso y construir un nuevo desarrollo (Madariaga, 2014). Al ser la resiliencia un proceso dinámico que se construye, la familia juega un rol sumamente importante, junto con la escuela y la comunidad (Fiorentino, 2008), por lo cual, estudiar esta relación es de gran importancia para fomentar el desarrollo de dimensiones que conformen un estilo parental que propicie hijos resilientes.

Han pasado más de cinco décadas desde que la psicología comenzó a centrarse más en los aspectos positivos del ser humano y a disminuir la atención hacia lo que, hasta ese momento, se consideraba como el objeto formal de estudio de esta ciencia: las enfermedades mentales. Es así que corrientes como el humanismo y la psicología positiva adquieren mayor importancia y, dentro de estas, la resiliencia es uno de los conceptos clave (Zakeri, Jowkar \& Razmjoee, 2010). Esta es definida de diversas formas, sin embargo, en casi todas ellas se hace referencia a la capacidad de hacer frente a los problemas de una manera positiva. Una definición más técnica es la de forma consensual, como los patrones de adaptación positiva en un contexto de riesgo y adversidad (Fernandes de Araújo, Teva \& Bermúdez, 2015).

Esta aptitud de muchas personas que han sufrido situaciones indeseables para llevar unas vidas normales es un testimonio claro de la elasticidad del espíritu humano y de su capacidad de resiliencia. Los que han tenido que superar una gran prueba describen el encuentro con una persona significativa, la comprensión, el amor, la fantasía, el optimismo, el humor, el afecto, la aceptación de sí mismo, la 
ilusión, la alegría, la generosidad, la esperanza, la creatividad, el gusto por la vida, el trabajo, etc., como factores de resiliencia (Muñoz, 2005). Por lo tanto, podemos decir que la resiliencia no es un fenómeno tan extraño y escaso como se creía inicialmente, sino que muchas veces forma parte de la vida cotidiana, distinguiéndose así cuatro dimensiones en la adaptación de la Escala de Resiliencia de Wagnild y Young, para Perú: Interés, Energía y esfuerzo, Identidad de la finalidad y Decisión (Castilla, Caycho, Shimabukuro, Valdivia \& Torres-Calderón, 2014).

Si bien el concepto de resiliencia se introduce en la psicología en la década de los ochenta, a partir de los estudios de Werner y Smith en 1982 (citados en Uriarte, 2005), es desde mucho antes que, con trabajos de Spitz y Bowlby (citados en Madariaga, 2014), se comenzó a evaluar a individuos que, inexplicablemente, se sobreponían, e incluso potenciaban sus capacidades, en entornos sumamente desfavorables. Es precisamente este último autor, pionero en la teoría del apego, quien relaciona la importancia del vínculo familiar con el desarrollo de la resiliencia. A esta línea apuntan autores como Mikulinar y Florian (1998, citados en Acero, 2009), quienes afirman que ciertos modos de interacción entre el niño y su cuidador principal durante la infancia temprana contribuyen notablemente al desarrollo de esta capacidad. Siendo que el apego está determinado por el estilo parental con el que los padres se interrelacionan con sus hijos, es que autores como Vanistendael y sus colaboradores (2009) proponen un modelo de construcción de la resiliencia a partir de factores que van íntimamente relacionados con este estilo, de lo cual se deduce que es el modelo formativo de los padres el que, en gran medida, propiciará el desarrollo de lo que conocemos como inmunología psíquica (Madariaga, 2014).

Desde hace más de 20 años, diversas investigaciones confirman la influencia de los estilos parentales en la salud mental de los adolescentes, de manera que los padres facilitan el ajuste positivo de sus hijos cuando estos son expuestos a amenazas y condiciones estresantes, utilizando apoyo emocional y conductual (Zakeri et al., 2010). Así, decimos que la adolescencia es un periodo en el cual la persona pasa por veloces transformaciones fisiológicas, cognitivas y emocionales, donde el desarrollo normal de estas áreas se ve afectado en gran medida por el interés parental y participación emocional, al igual que los estilos parentales (Buresová, Bartosova \& Cernak, 2015).

Diversos autores enfocados al estudio de la familia, y en saber cómo se desarrollan los individuos dentro de estas, han clasificado el conjunto de conductas que se manifiestan en el proceso de crianza de los hijos en lo que denominamos estilos parentales, siendo Diana Baumrind (1991) una de las más influyentes. Ella estableció cuatro tipos de estilos en base a las dimensiones de Control y Afecto, y reconoció que en la adolescencia se pueden identificar hasta siete tipos de estilos parentales: 
autoritativo, democrático, directivo, autoritario directivo y no autoritario directivo, suficiente, no directivo y rechazante. Además, mencionó que los adolescentes de familias autoritativas y democráticas eran competentes, independientes, maduros, resilientes, optimistas y percibían a sus padres como amorosos e influyentes.

Actualmente, dentro de todas las capacidades que se desarrollan, dependiendo del estilo parental en base al que se fue educado, es la resiliencia una de las que más ha capturado la atención; de manera que diversas investigaciones confirman que los factores familiares juegan un rol importante en el desarrollo del niño: una crianza efectiva ayuda a que el niño desarrolle resiliencia, al igual que media directamente copiando respuestas en la pubertad, enfermedades, dolor ante la pérdida de un ser querido y violencia comunitaria (Yakoh, Chongrukasa \& Prinyapol, 2015). A esto se añade la propuesta de Oliva y sus colaboradores, quienes postulan que el estilo parental es un concepto multidimensional y que, más allá del afecto y el control, existen otras variables que deberían ser tenidas en cuenta, por lo cual, se deben considerar las siguientes seis dimensiones: 1) Afecto y Comunicación, 2) Promoción de autonomía, 3) Control conductual, 4) Control psicológico, 5) Revelación y 6) Humor. Así, se puede hablar de un buen estilo parental como el poseedor de estas dimensiones (con excepción de una) y no de un determinado estilo de crianza, dentro de las clasificaciones conocidas (Oliva, Parra \& Arranz, 2008).

A esto se suma la importancia de analizar algunos factores, como el sexo del evaluado, dado que los varones han demostrado tener menor monitoreo parental y mayor permisividad, al igual que menor orientación, por parte de sus padres, a diferencia de las mujeres (Okorodudu, 2010). También, las relaciones que mantienen los adolescentes con los padres dependen de muchas variables, entre ellas, ser padre o madre, pero también de la forma en que el adolescente los percibe a cada uno, ya que, en función de esto, él se comunica con sus padres (Montañés Sánchez, Bartolomé Gutiérrez, Montañés Rodríguez \& Parra Casado, 2008). De igual manera, se debe tener en cuenta la presencia de este a lo largo del tiempo, es decir, si actualmente el adolescente vive con la(s) persona(s) con quien(es) se crió, debido a que los cambios en la familia, en las interacciones y en las pautas de crianza pueden afectar los estilos parentales y el desarrollo socioafectivo en la infancia (Cuervo, 2010).

Basándonos en lo anteriormente señalado y en las dimensiones trabajadas por Oliva y sus colaboradores (2008), nuestro objetivo general es identificar la relación entre estilos parentales y resiliencia. Para lo cual, tenemos objetivos específicos, tales como: (a) examinar los niveles encontrados en las dimensiones, tanto de estilos parentales como resiliencia; (b) identificar la relación entre las dimensiones de estilos parentales con las de resiliencia, y (c) finalmente, identificar las medias de las dimensiones según el sexo de la muestra. 


\section{Método}

El presente es un estudio empírico asociativo explicativo con un muestreo no aleatorio transversal (Ato, López \& Benavente, 2013).

\section{Participantes}

El muestreo fue no probabilístico de tipo intencional. La muestra estuvo conformada por 201 estudiantes: 72 varones y 128 mujeres, de 16 a 19 años $(X=17,7$; D.T.= $\mathrm{o}, 87$ ), de diez carreras distintas, todos con un grado de instrucción técnico, solteros y que cursaban el I, II y III semestre en un instituto de educación superior de Arequipa. De los cuales, el 69,2 \% se crio con ambos padres y el 51,7 \% sigue viviendo con ellos.

\section{Instrumentos}

La Escala para la Evaluación del Estilo Parental (Oliva, Parra, Sánchez \& López, 2007) es una escala de autorreporte que sirve para evaluar la percepción que tiene el adolescente de diversas dimensiones del estilo educativo de ambos padres; se puede aplicar de forma individual o colectiva a adolescentes a partir de los 12 años.

Está compuesta de 41 ítems de tipo cerrado construidos según una escala de tipo Likert de seis alternativas comprendidas entre 1 (Totalmente en desacuerdo) y 6 (Totalmente de acuerdo). Los ítems se agrupan en seis dimensiones: 1) Afecto y comunicación, que evalúa la expresión de apoyo y afecto por parte de los padres, su disponibilidad y la fluidez en su comunicación; 2) Promoción de autonomía, evalúa en qué medida los padres animan a su hijo para que tenga sus propias ideas y tome sus propias decisiones; 3 ) Control conductual, se refiere al establecimiento de límites y a los intentos de los padres por mantenerse informados sobre el comportamiento de los hijos fuera de casa; 4) Control psicológico, se refiere a la utilización por parte de los padres de estrategias manipuladoras; 5) Revelación, evalúa la frecuencia con la que el adolescente cuenta por iniciativa propia sus asuntos personales; 6) Humor, evalúa en qué medida el adolescente considera que sus padres muestran optimismo y buen sentido del humor. En el presente estudio, la consistencia interna, evaluada a través del Alfa de Cronbach, ha sido adecuada a nivel general $(\alpha=0,75)$, y muy buena, en las distintas dimensiones $(1, \alpha=0,929 ; 2$, $\alpha=0,854 ; 3, \alpha=0,883 ; 4, \alpha=0,925 ; 5, \alpha=0,934 ; 6, \alpha=0,902)$.

La Escala de Resiliencia de Wagnild y Young (1993) evalúa el nivel de resiliencia del individuo. Puede ser aplicada a adolescentes y adultos, aunque es posible utilizarla también con personas mayores, y puede administrarse de forma individual o 
colectiva. La escala está compuesta por 25 ítems de tipo Likert con seis alternativas cuya valoración está comprendida entre 1 (Totalmente en desacuerdo) y 6 (Totalmente de acuerdo). Se divide en cuatro factores: 1) Interés, 2) Energía y esfuerzo, 3) Identidad de la finalidad y 4) Decisión. Dicha escala ha sido adaptada y validada en Perú (Lima) por Castilla y colaboradores (2014). En este estudio, mediante el Alfa de Cronbach se obtuvo la consistencia interna, siendo la de la escala a nivel general $(\alpha=0,73)$, y las de sus distintas dimensiones: $1, \alpha=0,701 ; 2, \alpha=0,437 ; 3, \alpha=0,622 ; 4$, $\alpha=0,486$.

También, se recolectaron los datos sociodemográficos de los individuos a través de un breve cuestionario donde los participantes llenaron sus datos personales, tales como edad, sexo, grado de instrucción, ocupación, carrera, semestre y estado civil. Además, se agregaron tres preguntas de tipo cerrado. La primera tiene como afirmación «Me crie con» y cuatro alternativas: Padre, Madre, Ambos y Otros. La segunda dice «Actualmente vivo con» y tiene cinco alternativas: Solo, Padre, Madre, Ambos y Otros. Finalmente, la tercera corresponde a «si trabaja o no», teniendo para ello dos opciones: Sí o No.

\section{Procedimiento}

Los instrumentos se administraron en forma colectiva, en las aulas de clase del instituto y dentro del horario académico de los estudiantes. Se brindaron las instrucciones pertinentes, teniendo en consideración las normas éticas del anonimato y la confidencialidad. Previamente, se realizaron las gestiones pertinentes para obtener las autorizaciones respectivas del director del instituto y de los profesores.

\section{Análisis de datos}

Primero, se realizó una tabulación de las dimensiones, tanto de estilos parentales como de resiliencia, al igual que el total de cada una de ellas, para esto se obtuvieron percentiles según la muestra analizada y, en base a ello, se pudieron clasificar las puntuaciones obtenidas en los tres niveles (bajo, medio y alto), como indica la prueba utilizada (ver Tablas 1 y 2). Posteriormente, se realizó un análisis de correlaciones de la edad, las dimensiones de estilos parentales, así como de resiliencia, a través de una correlación de Pearson. Finalmente, se compararon las medias entre las dimensiones de estilos parentales y resiliencia según el sexo de la muestra, para lo cual se utilizó la prueba t de Student. La codificación y el análisis de datos se realizaron con el paquete estadístico SPSS versión 23. 


\section{Resultados}

Conforme a lo encontrado en las puntuaciones finales de los estilos parentales (ver Tabla 1), se tiene que, en general, el 5o,7 \% de la muestra tiene un estilo parental promedio (ni alto ni bajo).

En cuanto a Afecto y Comunicación, en el $23 \%$ no está muy presente, en el 53,2 \% parece encontrarse de forma regular y en el $23,4 \%$ se encuentra muy presente. En cuanto a Control conductual, en el $24,4 \%$ no está muy presente, en el 54,2 \% parece encontrase de forma regular y en el 21,4 \% está muy presente. En cuanto a Control psicológico, en el 22,9 \% no está muy presente, en el 53,2 \% parece encontrase de forma regular y en el 23,9\% se encuentra muy presente. En cuanto a Promoción de autonomía, en el $23,9 \%$ no está muy presente, en el 55,7 \% parece encontrase de forma regular y en el 20,4\% se encuentra muy presente. En cuanto a Humor, en el 22,9\% no está muy presente, en el 52,2 \% parece encontrase de forma regular y en el $24,9 \%$ se encuentra muy presente. En cuanto a Revelación, en el $22,9 \%$ no está muy presente, en el 53,2 \% parece encontrase de forma regular y en el 23,9\% se encuentra muy presente.

\section{Tabla 1.}

Estilo parental

\begin{tabular}{|l|l|l|l|}
\hline & Nivel & N & $\%$ \\
\hline \multirow{4}{*}{ Afecto y comunicación } & Bajo & 47 & 23,4 \\
\cline { 2 - 4 } & Medio & 107 & 53,2 \\
\cline { 2 - 4 } & Alto & 47 & 23,4 \\
\hline Control conductual & Bajo & 49 & 24,4 \\
\cline { 2 - 4 } & Medio & 109 & 54,2 \\
\cline { 2 - 4 } & Alto & 43 & 21,4 \\
\hline Control psicológico & Bajo & 46 & 22,9 \\
\cline { 2 - 4 } & Medio & 107 & 53,2 \\
\cline { 2 - 4 } & Alto & 48 & 23,9 \\
\hline Promoción de autonomía & Bajo & 48 & 23,9 \\
\cline { 2 - 4 } & Medio & 112 & 55,7 \\
\cline { 2 - 4 } & Alto & 41 & 20,4 \\
\hline \multirow{5}{*}{ Humor } & Bajo & 46 & 22,9 \\
\cline { 2 - 4 } & Medio & 105 & 52,2 \\
\cline { 2 - 4 } & Alto & 50 & 24,9 \\
\hline
\end{tabular}




\begin{tabular}{|l|l|l|l|}
\hline \multirow{4}{*}{ Revelación } & Bajo & 44 & 21,9 \\
\cline { 2 - 4 } & Medio & 107 & 53,2 \\
\cline { 2 - 4 } & Alto & 50 & 24.9 \\
\hline \multirow{3}{*}{ Total de estilo parental } & Bajo & 49 & 24,4 \\
\cline { 2 - 4 } & Medio & 102 & 50,7 \\
\cline { 2 - 4 } & Alto & 50 & 24,9 \\
\hline
\end{tabular}

Por otro lado, en relación a las puntuaciones finales encontradas en resiliencia (ver Tabla 2), podemos observar que apenas más de la mitad de la muestra, el 51,7 \%, obtuvo un nivel de resiliencia medio, mientras que el $24,4 \%$ obtuvo un nivel bajo y solo el 23,9\% de los estudiantes encuestados tuvieron alto nivel de resiliencia. En las cuatro dimensiones de la resiliencia se obtuvieron porcentajes muy similares en los respectivos niveles: bajo, medio y alto. Es decir, todas las dimensiones siguieron el mismo patrón.

Se observó un mayor porcentaje de nivel bajo de resiliencia en la dimensión Interés, con un $24,4 \%$; la dimensión con mayor porcentaje de nivel medio de resiliencia fue Identificación de la Finalidad, con un $61,2 \%$, y la dimensión que obtuvo mayor porcentaje en un nivel alto de resiliencia fue Decisión, con un 22,4\%.

\section{Tabla 2.}

Resiliencia

\begin{tabular}{|l|l|l|l|}
\hline & Nivel & N & $\%$ \\
\hline \multirow{5}{*}{ Interés } & Bajo & 49 & 24,4 \\
\cline { 2 - 4 } & Medio & 108 & 53,7 \\
\cline { 2 - 4 } & Alto & 44 & 21,9 \\
\hline \multirow{5}{*}{ Idenergía y esfuerzo } & Bajo & 46 & 22,9 \\
\cline { 2 - 4 } & Medio & 113 & 56,2 \\
\cline { 2 - 4 } & Alto & 42 & 20,9 \\
\hline \multirow{5}{*}{ Decisión } & Bajo & 35 & 17,4 \\
\cline { 2 - 4 } & Medio & 123 & 61,2 \\
\cline { 2 - 4 } & Alto & 43 & 21,4 \\
\hline \multirow{5}{*}{ Total de resiliencia de la finalidad } & Bajo & 42 & 20,9 \\
\cline { 2 - 4 } & Medio & 114 & 56,7 \\
\cline { 2 - 4 } & Alto & 45 & 22,4 \\
\hline & Bajo & 49 & 24,4 \\
\cline { 2 - 4 } & Medio & 104 & 51,7 \\
\cline { 2 - 4 } & Alto & 48 & 23,9 \\
\hline
\end{tabular}


En cuanto a las correlaciones, se aplicó la prueba de Pearson para valorar las relaciones entre las dimensiones de estilos parentales y las dimensiones de resiliencia (ver Tabla 3), siendo la mayor parte de estas significativas y positivas. Así, entre las correlaciones positivas y significativas tenemos la relación de Afecto y Comunicación, Promoción de autonomía, Humor, Revelación, y el puntaje total de estilo parental con Interés, Energía y esfuerzo, Identidad de la finalidad y Decisión.

\section{Tabla 3.}

Correlación de Pearson entre la edad, estilo parental y resiliencia

\begin{tabular}{|c|c|c|c|c|c|c|c|c|c|c|c|c|c|}
\hline & 1 & 2 & 3 & 4 & 5 & 6 & 7 & 8 & 9 & 10 & 11 & 12 & 13 \\
\hline 1.ED & 1 & ,095 & ,095 & ,095 & , 095 & ,095 & , 095 & , 095 & , 095 & ,095 & ,095 & ,095 & ,095 \\
\hline 2.AC & & 1 & $483^{* *}$ & $264^{* *}$ &, $733^{* *}$ & $849^{* *}$ &, $722^{\text {*kt }}$ & , $908^{* *}$ & ,116 &, $222^{*}$ & , $218^{* *}$ & , $142^{*}$ & , 199 \\
\hline 3.CC & & & 1 &,$- 255^{* *}$ &, $393^{* *}$ & , $487^{* *}$ &, $411^{* *}$ &, $512^{* *}$ & ,208 & ,117 & , 072 & , 055 & , 073 \\
\hline 4.CP & & & & 1 &, $306^{* *}$ & ,274 & , $167^{*}$ &, $452^{* *}$ & -,016 & , 025 & , &,- 056 & , 005 \\
\hline 5.PA & & & & & 1 &, $730^{* * *}$ & $645^{* *}$ & $863^{* *}$ & 138 & ,209** & , 247 & ,200 &, $226^{\text {htx }}$ \\
\hline 6.HUM & & & & & & 1 &, $724^{* * *}$ & ,904 & , 084 & ,207 & , 224 & , 147 & $183^{*}$ \\
\hline 7.REV & & & & & & & 1 & $806^{* *}$ & ,161" & ,204 & ,291 & , 142 &, $236^{\text {th }}$ \\
\hline 8.TEP & & & & & & & & 1 & 114 & , $218^{* *}$ &, $248^{* *}$ & ,139* & $205^{\text {t* }}$ \\
\hline 9.INT & & & & & & & & & 1 &, $561^{* *}$ & ,667 &, $545^{\text {*kx }}$ & $890^{* * *}$ \\
\hline 10.EF & & & & & & & & & & 1 &, $592^{* *}$ &, $589^{* * *}$ &, $784^{* * *}$ \\
\hline 11.IF & & & & & & & & & & & 1 & $627^{* *}$ & $865^{* * *}$ \\
\hline 12.DEC & & & & & & & & & & & & 1 &, $775^{* * *}$ \\
\hline 13.TR & & & & & & & & & & & & & 1 \\
\hline
\end{tabular}

Nota. $\mathrm{ED}=\mathrm{Edad} ; \mathrm{AC}=$ Afecto y Comunicación; $\mathrm{CC}=$ Control conductual; $\mathrm{CP}=$ Control psicológico; $\mathrm{PA}=$ Promoción de autonomía; $\mathrm{HUM}=\mathrm{Humor} ; \mathrm{REV}=$ Revelación; $\mathrm{TEP}=$ Total de Estilos Parentales; INT= Interés; EF= Energía y esfuerzo; IF= Identidad de la finalidad; DEC= Decisión; TR= Total de Resiliencia.

${ }^{*} \mathrm{p}<, 05 ;{ }^{* *} \mathrm{p}<, 01$

Y por último, sobre la comparación de medias realizadas a través de la prueba paramétrica t de Student según el sexo (ver Tabla 4), se encontró que las dimensiones del estilo parental evaluadas que tienen la puntuación media más alta son Afecto y Comunicación $(X=33,1)$, en varones, y Promoción de autonomía $(X=36,1)$, en mujeres; mientras que la dimensión del estilo parental Revelación tiene la puntuación media más baja, tanto en varones $(X=17,0)$ como en mujeres $(X=19,7)$. Asimismo, el valor promedio de la puntuación total del estilo parental para la muestra estudiada fue de 166,5 , para varones, y de 176,4 , para mujeres. 
Tabla 4.

Comparación de medias entre estilo parental y resiliencia según el sexo

\begin{tabular}{|c|c|c|c|c|c|c|}
\hline & Sexo & $\mathbf{N}$ & X & D.T & $\mathbf{t}$ & $\mathbf{p}$ \\
\hline \multirow[t]{2}{*}{ AC } & Varón & 72 & 33,1 & 9,9 & $-1,423$ & 0,156 \\
\hline & Mujer & 128 & 35,1 & 8,9 & $-1,384$ & 0,168 \\
\hline \multirow[t]{2}{*}{ CC } & Varón & 72 & 26,8 & 7,3 & $-2,808$ & $0,005^{* *}$ \\
\hline & Mujer & 128 & 29,4 & 5,9 & $-2,654$ & $0,009^{*}$ \\
\hline \multirow[t]{2}{*}{$\mathrm{CP}$} & Varón & 72 & 31,9 & 9,8 & 1,606 & 0,110 \\
\hline & Mujer & 128 & 29,7 & 9,5 & 1,593 & 0,113 \\
\hline \multirow[t]{2}{*}{ PA } & Varón & 72 & 33,0 & 10,1 & $-2,301$ & $0,022^{*}$ \\
\hline & Mujer & 128 & 36,1 & 8,6 & $-2,198$ & $0,030^{*}$ \\
\hline \multirow[t]{2}{*}{ HUM } & Varón & 72 & 24,5 & 7,8 & $-1,605$ & 0,110 \\
\hline & Mujer & 128 & 26,3 & 7,2 & $-1,570$ & 0,119 \\
\hline \multirow[t]{2}{*}{ REV } & Varón & 72 & 17,0 & 6,9 & $-2,600$ & $0,010^{* *}$ \\
\hline & Mujer & 128 & 19,7 & 7,1 & $-2,625$ & $0,010^{* *}$ \\
\hline \multirow[t]{2}{*}{ TEP } & Varón & 72 & 166,5 & 38,9 & $-1,840$ & 0,067 \\
\hline & Mujer & 128 & 176,4 & 35,1 & $-1,788$ & o,o76 \\
\hline \multirow[t]{2}{*}{ INT } & Varón & 72 & 46,1 & 6,6 & 0,942 & 0,384 \\
\hline & Mujer & 128 & 45,2 & 6,3 & 0,929 & 0,354 \\
\hline \multirow[t]{2}{*}{ EF } & Varón & 72 & 23,6 & 3,5 & $-0,344$ & 0,731 \\
\hline & Mujer & 128 & 23,8 & 3,3 & $-0,338$ & 0,736 \\
\hline \multirow[t]{2}{*}{ IF } & Varón & 72 & 27,9 & 4,2 & $-0,283$ & 0,777 \\
\hline & Mujer & 128 & 28,1 & 4,4 & $-0,286$ & 0,775 \\
\hline \multirow[t]{2}{*}{ DEC } & Varón & 72 & 18,5 & 3,1 & $-0,064$ & 0,949 \\
\hline & Mujer & 128 & 18,5 & 2,9 & $-0,062$ & 0,950 \\
\hline \multirow[t]{2}{*}{ TR } & Varón & 72 & 166,1 & 14,9 & 0,240 & 0,810 \\
\hline & Mujer & 128 & 115,6 & 14,2 & 0,237 & 0,813 \\
\hline
\end{tabular}

Nota. $\mathrm{AC}=$ Afecto y Comunicación; $\mathrm{CC}=$ Control conductual; $\mathrm{CP}=$ Control psicológico; $\mathrm{PA}=$ Promoción de autonomía; HUM $=$ Humor; $\mathrm{REV}=$ Revelación; TEP = Total de Estilos Parentales; $\mathrm{INT}=$ Interés; $\mathrm{EF}=$ Energía y esfuerzo; $\mathrm{IF}=$ Identidad de la finalidad; $\mathrm{DEC}=$ Decisión; $\mathrm{TR}=$ Total de Resiliencia. ${ }^{*} \mathrm{p}<, 05 ;{ }^{* *} \mathrm{p}<, 01$

Respecto a las dimensiones de la resiliencia evaluadas, se observa que la dimensión con puntuación media más alta es Interés, en varones $(X=46,1)$ y en mujeres $(X=45,2)$; mientras que la dimensión de la resiliencia Decisión tiene una puntua- 
ción media más baja, tanto en varones $(X=18,5)$ como en mujeres $(X=18,5)$. Asimismo, el valor promedio de la puntuación total de la resiliencia para la muestra estudiada fue de 166,1 para varones, y de 115,6, para mujeres. Sin embargo, las únicas diferencias significativas encontradas están en las dimensiones del estilo parental, tales como Control conductual, Revelación y Promoción de autonomía.

\section{Discusión}

La finalidad de este estudio era identificar si existe alguna relación entre el estilo parental y la resiliencia en adolescentes, para lo cual, se examinaron, en primer lugar, los niveles obtenidos en las dimensiones de estilos parentales como en las de resiliencia. Se encontró que, en estilo parental, los resultados se ubican en el nivel medio, en todas las dimensiones, de igual forma en el total general. Ello quiere decir que la muestra posee un estilo parental adecuado según la normalidad estadística.

Por otro lado, en cuanto a la resiliencia, al igual que el estilo parental, se identificó que la mayoría de los resultados obtenidos se encuentran en un nivel medio en las cuatro dimensiones y en el total general. Lo que indica que los sujetos no tienen definido un factor de resiliencia que predomina en ellos, que puede deducirse desde el poco conocimiento de sí mismos, tomando en cuenta la etapa de desarrollo (adolescencia-preadultez) y condiciones ambientales inestables (cambio de educación secundaria a educación superior) en la que se encuentran. Por ello, los resultados actuales deben de ser tomados desde este contexto (Buresová et al., 2015).

A su vez, se puede observar que existe correlación entre el estilo parental y resiliencia, si bien este índice no es muy alto, es bastante significativo para demostrar la relación entre variables. Con respecto a las dimensiones identificadas, se puede decir que la dimensión con mayor correlación es Afecto y Comunicación. La misma destaca en cuanto a las variables que inducen a la persona a la autonomía, tales como Promoción de autonomía, Humor, Revelación, Energía y esfuerzo e Identidad de la finalidad. En cuanto a la relación entre Afecto y Resiliencia, existe una correlación significativa entre ambas variables. Esto coincide con lo encontrado por Zakeri y colaboradores (2010), dado que el apoyo, afecto y un estilo parental centrado en el niño está asociado con el desarrollo de la resiliencia. Si bien Oliva et al. (2008) detalla tres estilos relacionales que engloban estas dimensiones, dichos estudios han sido realizados únicamente como parte de una investigación en Sevilla y Huelva (España), por lo que es necesario aplicar la escala a poblaciones más amplias y, de esta manera, establecer estilos relacionales de acuerdo a los resultados que se obtengan en estas, tal como lo hizo Gómez, Del Rey, Romera y Ortega (2015) en Córdoba. 
Por otro lado, las dimensiones que se identificaron con menor correlación son: Control conductual y Control psicológico, ya que muestran puntajes más bajos en relación con otros factores. Un aspecto que cabe mencionar es que el Control psicológico, descrito anteriormente, se encuentra en constante relación con todas las dimensiones identificadas en resiliencia, siendo este un indicador perjudicial para el desarrollo de la misma.

Y, por último, según los resultados mostrados en la comparación de medias, se ve que existen diferencias significativas entre varones y mujeres en las dimensiones de Control conductual, Promoción de autonomía y Revelación, lo cual puede deberse a las diferencias propias entre cada género y su connotación social. Esto coincide con lo mencionado por Okorodudu (2010), dado que las mujeres tienden a tener mayor Control conductual que los varones.

El resultado general de la investigación indica que sí existe correlación entre las variables estudiadas, y con ello se demuestra que el estilo parental tiene relación directa con la resiliencia que desarrollan los adolescentes. Así, podemos inferir que es posible trabajar con los padres en cuanto a las dimensiones de su estilo parental, para que puedan desarrollar con mayor fuerza y énfasis la resiliencia en sus hijos desde la infancia; tal como lo proponen Aguiar y Acle (2012), quienes resaltan la importancia de trabajar esta capacidad en la familia y en la escuela, fundamentalmente desde edades tempranas. Finalmente, es necesario profundizar en la investigación de otros factores que intervienen en el desarrollo de la resiliencia, considerando principalmente a la escuela (Fiorentino, 2008), la cual constituye otro gran eje en este proceso, debido al tiempo que los niños y adolescentes pasan en ella y a la gran influencia que ejerce a partir de la socialización con los pares y las enseñanzas de los docentes.

\section{Referencias}

Acero, P. (2009). La otra cara de la tragedia. Resiliencia y crecimiento postraumático. Buenos Aires: San Pablo Ediciones.

Aguiar, E., \& Acle, G. (2012). Resiliencia, factores de riesgo y protección en adolescente mayas de Yucatán: Elementos para favorecer la adaptación escolar. Acta Colombiana de Psicología, 15 (2), 53-64.

Ato, M., López, J., \& Benavente, A. (2013). Un sistema de clasificación de los diseños de investigación en psicología. Anales de Psicología, 29(3), 1038-1059.

Baumrind, D. (1991). The influence of parenting style on adolescent competence and substance use. Journal of Early Adolescence, 11(1), 56-95. 
Buresová, I., Bartosova, K., \& Cernak, M. (2015). Connection between parenting styles and self-harm in adolescence. Procedia. Social and Behavioral Sciences, 171, 1106-1113.

Castilla, H., Caycho, T., Shimabukuro, M., Valdivia, A., \& Torres-Calderón, M. (2014). Análisis exploratorio de la Escala de Resiliencia de Wagnild y Young en una muestra de adolescentes, jóvenes y adultos universitarios de Lima Metropolitana. Revista de Psicología de Arequipa, 5(1), 121-136.

Cuervo, A. (2010). Pautas de crianza y desarrollo socioafectivo en la infancia. Revista Diversitas. Perspectivas en Psicología, 6(1), 111-121.

Fernandes de Araújo, L., Teva, I., \& Bermúdez, M. (2015). Resiliencia en adultos: Una revisión teórica. Terapia Psicológica, 33(3), 257-276.

Fiorentino, M. (2008). La construcción de la resiliencia en el mejoramiento de la calidad de vida y la salud. Suma Psicológica, 15(1), 95-113.

Gómez, O., Del Rey, R., Romera, E., \& Ortega, R. (2015). Los estilos educativos paternos y maternos en la adolescencia y su relación con la resiliencia, el apego y la implicación en acoso escolar. Anales de Psicología, 31(3), 979-989.

Lozada, C. (2012). Deserción en institutos superiores llega al 6o \%. Diario Correo [Edición online]. Recuperado el 23 de mayo del 2016 de: http://diariocorreo. pe/ciudad/desercion-en-institutos-superiores-llega-al-6o-517310/

Madariaga, J. (2014). Nuevas miradas sobre la resiliencia. Barcelona: Gedisa.

Montañés Sánchez, M., Bartolomé Gutiérrez, R., Montañés Rodríguez, J., \& Parra Casado, M. (2008). Influencia del contexto familiar en las conductas adolescentes. Ensayos, 17(23), 391-407.

Muñoz, V. (2005). Educar para la resiliencia: Un cambio de mirada en la prevención de situaciones de riesgo social. Revista Complutense de Educación, 16(1), 107-124.

Okorodudu, G. (2010). Influence of parenting styles on adolescent delinquency in delta central senatorial district. Journal of Counselling, 3(1), 58-86.

Oliva, A., Parra, A., \& Arranz, E. (2008). Estilos relacionales parentales y ajuste adolescente. Infancia y Aprendizaje, 31(1), 93-106.

Oliva, A., Parra, A., Sánchez, I., \& López, F. (2007). Estilos educativos materno y paterno: Evaluación y relación con el ajuste adolescente. Anales de Psicología, 23(1), 49-56.

Uriarte, J. (2005). La resiliencia: Una nueva perspectiva en psicopatología del desarrollo. Revista de Psicodidáctica, 10(2), 61-79.

Vanistendael, E., Vilar, J., \& Pont, E. (2009). Reflexiones en torno a la resiliencia: Una conversación con Stefan Vanistendael. Educación Social, 43, 93-103. 
Villalta, M. (2009). Factores de resiliencia asociados al rendimiento académico en estudiantes de contextos de alta vulnerabilidad social. Revista de Pedagogía, 31(88), 159-188.

Wagnild, G. M., \& Young, H. M. (1993). Development and psychometric evaluation of the Resiliencie Scale. Journal of Nursing Measurement, 1(2), 165-178.

Yakoh, M., Chongrukasa, D., \& Prinyapol, P. (2015). Parenting styles and adversity quotient of youth at Pattani Foster home. Procedia. Social and Behavioral Sciences, 205, 282-286.

Zakeri, H., Jowkar, B., \& Razmjoee, M. (2010). Parenting styles and resilience. Procedia. Social and Behavioral Sciences, 5, 1067-1070. 